\title{
Propiedades psicométricas del Inventario Breve de Experiencias Óptimas (Flow)
}

\author{
Calero, Alejandra*1 \& Injoque-Ricle, Irene* \\ * Facultad de Psicología, Universidad de Buenos Aires. CONICET, Argentina.
}

\begin{abstract}
Resumen. El fluir (flow) refiere a situaciones en las que los sujetos se implican en actividades por motivación intrínseca experimentando en la realización un gran disfrute. El objetivo de este trabajo es presentar la validación del Inventario Breve de Experiencias Óptimas en una muestra de adolescentes. Para ello se les administró el Inventario Breve de Experiencias Óptimas junto con medidas externas a 211 adolescentes escolarizados de la Ciudad autónoma de Buenos Aires, Argentina, con una media 14.26 años $(D E=1.21)$. El inventario está compuesto por 9 ítems, a partir de los cuales se obtiene un puntaje total de experiencia óptima en la actividad evaluada. Se analizaron las propiedades psicométicas del instrumento, estimando la consistencia interna y las evidencias de validez. Los resultados permiten concluir que el inventario es válido y confiable para medir las experiencias óptimas en adolescentes escolarizados de la Ciudad Autónoma de Buenos Aires, Argentina. Palabras clave: Experiencia óptima - Actividades - Adolescentes - Validación
\end{abstract}

\section{Psychometric properties of the Optimal Experiences Brief Inventory (Flow)}

\begin{abstract}
The optimal experience or Flow refers to situations in which individuals are engaged in activities for intrinsic motivation in achieving great enjoyment. The aim of this work is to present the validation of the Brief Inventory of Optimal Experiences in a sample of adolescents. The Brief Inverntory of Optimal Experiences, along with external measures was administered to a sample of 211 adolescent from the Autonomous City of Buenos Aires, Argentina. They had with a mean age of 14.26 years $(S D=1.21)$. The inventory includes 9 items, from which a total score of optimal experience is obtained. The psychometric properties of the instrument were analyzed by estimating the internal consistency and looking for evidence of validity. The results showed that the Brief Inventory of Optimal Experiences is valid and reliable instrument to assess optimal experiences (flow) among adolescent from the Autonomous City of Buenos Aires, Argentina.

Key Words: Optimal Experience - Activities - Adolescent - Validation.
\end{abstract}

\section{Introducción}

El término fluir (flow) o experiencia óptima emergió a partir de las descripciones de la experiencia subjetiva de estas personas como una vivencia que se produce de una forma no pasiva pero sin esfuerzo aparente. Este concepto tiene su origen en el estudio de las experiencias positivas de los sujetos al involucrarse en actividades sin obtener otro beneficio

1 Esta investigación fue financiada por el Consejo Nacional de Investigaciones Científicas y Técnicas (CONICET Res. Nº02314/10) y Por la Secretaría de Ciencia y Técnica de la Universidad de Buenos Aires (UBACyT 200201001 00052). Beca interna de postgrado Tipo 1, CONICET, "Experiencias positivas -flow- en las actividades escolares y extraescolares durante la adolescencia. Su relación con el autoconcepto y las conductas de riesgo". Directora: Leibovich de Figueroa, N. Investigación Proyecto UBACYT 052 "Ecoevaluacion psicológica de la interfaz adolescente-adulto. Contribución de las experiencias óptimas (flow)". Directora: Leibovich de Figueroa N. Codirectora: Schmidt, Vanina. Dirección de contacto: acalero@psi.uba.ar. Juan Lavalle2353. 
que la tarea en sí misma. Este desarrollo refiere a aquellas situaciones en las que un individuo, al involucrarse en determinadas actividades, logra poner la atención al servicio de cumplir un objetivo que es compatible con sus habilidades, siendo la actividad regulada por normas que brindan una retroalimentación inmediata sobre el desempeño. En estas situaciones los sujetos tienen la sensación de tener control sobre las acciones propias y sobre el entorno inmediato, son absorbidos por la experiencia produciéndose una pérdida de la noción del tiempo y del sí mismo como seres diferenciados de la tarea que se lleva adelante. Se define por un profundo sentimiento de disfrute. La actividad produce experiencias tan agradables que las personas se involucran por motivación intrínseca; la experiencia, por definición, es una recompensa en sí misma (Csikszentmihalyi \& Csikszentmihalyi, 1998).

La experiencia óptima -flow-, según Csikszentmihalyi, se compone de nueve dimensiones que la caracterizan (Csikszentmihalyi, 1990; Csikszentmihalyi \& Csikszentmihalyi, 1998): Equilibrio entre la habilidad percibida y el desafío que plantea la actividad, ocurre cuando la habilidad percibida está al nivel adecuado para enfrentarse a las demandas de la situación, que están por encima del común/promedio la persona; Unión entre la acción y la conciencia, explica el compromiso y la absorción total en la actividad, la cual es tan profunda que las acciones se vuelven espontáneas o automáticas; Metas claras, permite a la persona una fuerte sensación de qué es lo que quiere conseguir con la tarea; Retroalimentación clara y directa, le permite a la persona saber que se está logrando su objetivo; Concentración en la tarea presente, permite que nada pueda correr el foco de la persona de la tarea que está realizando; Sentimiento o percepción de control sobre la tarea que se está realizando, aún cuando la tarea sea muy difícil; Pérdida de la autoconciencia, que es la desaparición de la preocupación por la propia imagen o la opinión de los demás a medida que la persona se involucra en la actividad que está realizando; Deformación en la percepción del tiempo, puede llevar a la sensación de que las acciones se dan como en cámara lenta o, al contrario, que se pasan rápidamente; Experiencia autotélica, la experiencia tiene una finalidad en sí misma, que es el elemento clave de una experiencia óptima, y se refiere a una actividad que se realiza no por conseguir algún beneficio, sino simplemente porque hacerla, en sí, es la recompensa.

Este concepto se anida en los cimientos conceptuales de la psicología positiva. La psicología positiva es definida como el estudio científico de las experiencias positivas, de los rasgos individuales positivos, de las instituciones que facilitan su desarrollo y de los 
programas que ayudan a mejorar la calidad de vida de los individuos (Seligman \& Csikszentmihalyi, 2000). Este nuevo enfoque propone un cambio de mirada desde la psicopatología hacia aquellas características de las personas y sus entornos que promueven bienestar y satisfacción vital. Es en el contexto del estudio de las experiencias positivas, que este nuevo enfoque plantea, donde surge la importancia del concepto de experiencia óptima (flow).

El concepto experiencia óptima ha sido aplicado para el estudio de las experiencias positivas en las actividades que las personas de diferentes grupos etáreos se involucran. Se han realizado investigaciones en actividades como el deporte (García, Cervelló, Jiménez, Iglesias, \& Santos-Rosa, 2005; S. Jackson \& Marsh, 1996; Jackson, Kimiecik, Ford, \& Marsh, 1998; Moreno, Alonso, Martínez Galindo, \& Cervelló, 2005; Moreno Murcia, Cervelló Gimeno, \& González-Cutre, 2008), el arte (Bakker, 2005; Fritz \& Avsec, 2007; Roberts, Walton, \& Bogg, 2005), el trabajo (Csikszentmihalyi \& LeFevre, 1989; Chu, 2010; Leibovich, 2009; Salanova, Bakker, \& Llorens, 2006; Salanova, Martínez, Cifre, \& Schaufeli, 2005), actividades escolares (Csikszentmihalyi \& Larson, 1984; Shernoff, Csikszentmihalyi, Schneider, \& Shernoff, 2003; Shernoff \& Vandell, 2007) actividades utilizando tecnologías de informática y comunicación (Finneran \& Zhang, 2005; Ghani \& Deshpande, 1994; Rodríguez-Sánchez, Schaufeli, Salanova, \& Cifre, 2008), entre otras. A su vez, este constructo ha sido relacionado con otros conceptos relevantes en la Psicología como el autoconcepto (Jackson, Thomas, Marsh, \& Smethurst, 2001), la motivación (Keller, 2008; Martin et al., 2010; Moreno et al., 2005; Seifert \& Hedderson, 2010) y la ansiedad (López Torres, Torregosa, \& Roca, 2007).

La forma en la cual las experiencias óptimas se evaluaron desde su concepción abarca desde métodos cualitativos a cuantitativos. En un primer momento el estudio de la experiencia óptima se realizaba por medio de entrevistas y cuestionarios. Luego para alcanzar una mayor precisión en la medición de la calidad de la experiencia subjetiva, se desarrolló el Método de Muestreo de la Experiencia (MCsikszentmihalyi, 1990). Este método consiste en proporcionar una hoja de registro y un receptor electrónico. Los sujetos recibían entre 7 u 8 señales al azar a los largo del día y debían completar el registro en base a la actividad que estaban realizando en ese momento (Csikszentmihalyi, Larson, \& Prescott, 1977). A pesar de la riqueza de este instrumento, dado que evalúa la experiencia in situ, su utilización se ve dificultada debido a lo costoso de su implementación, tanto por la tecnología 
requerida para su evaluación, como para el procesamiento de los datos (Kimiecik, 2002). Posteriormente se desarrollaron numerosas escalas psicométricas autoinformadas para su evaluación (A.B. Bakker, 2008; Fournier et al., 2007; García Calvo, Jiménez Castuera, Santos-Rosa Ruano, Reina Vaíllo, \& Cervelló Gimeno, 2008; Mesurado, 2008; RodríguezSánchez et al., 2008; Salanova et al., 2005).

Entre las escalas más importantes que toman el modelo de experiencia óptima compuesto por las nueve dimensiones planteadas por Cziksentmihalyi, se destaca la Flow State Scale en sus diferentes versiones (Fournier et al., 2007; García Calvo et al., 2008; Jackson \& Marsh, 1996; Jackson, Martin, \& Eklund, 2008; Jackson \& Eklund, 2002). Esta escala está compuesta por 36 ítems (4 ítems por cada dimensión), que se agrupan en un factor principal y nueve factores secundarios. En un comienzo fue creada para evaluar las experiencias óptimas (flow) en las actividades deportivas, pero ha mostrado ser efectiva para evaluar la experiencia óptima en otros contextos (Jackson et al., 2008). En la actualidad esta escala cuenta con dos versiones, una que evalúa el estado de flow (Flow State Scale) y otra la disposición a experimentarlo (Dispositional Flow Scale) (Jackson et al., 1998). La escala ha sido adaptada en España (García Calvo et al., 2008), Francia (Fournier et al., 2007) y Portugal (Gouveia, Pais-Ribeiro, Moreira Marques, \& Carvalho, 2012), entre otros. Luego se construyeron versiones reducidas de las dos escalas, las cuales están compuestas por nueve ítems, cada uno de los reactivos que la componen representa una de las nueve dimensiones de la experiencia óptima. La idea de crear esta escala surgió por cuestiones pragmáticas, para aquellas situaciones en las que aplicar la versión extensa era dificultoso. Estas versiones de 9 ítems, a diferencia de las versiones de 36 ítems, tienen como objetivo evaluar solo el flow global que los sujetos experimentan en las distintas actividades, planteando la existencia de un modelo con un único factor latente sin los factores secundarios propios de las versiones extensas de la escala (Jackson et al., 2008).

El objetivo del presente artículo es generar una escala breve válida y confiable para evaluar las experiencias óptimas en adolescentes del área metropolitana de Buenos Aires, Argentina. Dicha escala estará basada en la adaptación española de la Flow State Scale (Escala de estado de fluidez) (García Calvo et al., 2008), en la Short Flow Scale (Jackson et al., 2008) y en ítems desarrollados localmente; a partir de los cuales de seleccionarán los que mejor se ajusten a nivel psicométrico y conceptual. Se espera lograr un inventario con una estructura factorial con un único factor latente. Y, finalmente, se pretende lograr obtener un 
inventario que logre evaluar las experiencias óptimas en la realización de diversas actividades en que los adolecentes participan.

\section{Método}

\section{Participantes}

La muestra estuvo compuesta por 211 adolescentes (151 mujeres $-71.6 \%$ - y 60 varones), con una edad media de 14.26 años $(D E=1.21)$ concurrentes a una escuela de educación media de la Ciudad Autónoma de Buenos Aires, Argentina. La escuela desde donde se extrajeron los casos corresponden a un estrato socioeconómico medio. Todos los sujetos participaron previa autorización de los padres a los cuales se les aseguró la confidencialidad de la información y el anonimato de los participantes.

\section{Instrumentos}

Desarrollo del Inventario: Se generó inicialmente una base de 50 ítems que operacionalizan las 9 dimensiones del Flow planteadas por Csikszentmihalyi (1990, 1998). En esta base inicial se encontraban ítems pertenecientes a la versión breve de Flow State Scale (S. Jackson et al., 2008), Escala de estado de fluidez (García Calvo et al., 2008) e ítems generados a partir de entrevistas realizadas a adolescentes. En el caso de los ítems de la escala de Jackson y colaboradores (2008), se realizó la adaptación lingüística al castellano rioplantense a través de un proceso de traducción y retraducción. Para ello se trabajó con dos grupos de psicólogos con excelente manejo del idioma inglés: el primero tradujo del inglés al castellano los ítems originales, y el segundo tradujo del castellano al inglés los ítems resultantes. La versión re-traducida fue evaluada y comparada con la versión original por parte de los psicólogos que realizaron la traducción inglés-castellano, encontrando que los ítems ambos grupos contaban con equivalencia conceptual.

En cuanto a los ítems de la adaptación española se realizó una adaptación lingüística a nuestro medio.

Por último, los ítems surgidos a partir de las entrevistas se generaron teniendo en cuenta la descripción que los adolescentes realizaron de lo que experimentaban al implicarse en una actividad que les generaba disfrute. 


\section{Procedimiento}

En primer lugar se realizó una depuración del pool inicial de 50 ítems por medio del criterio de jueces expertos a quienes se les solicitó que atiendan al contenido de cada uno de los reactivos en función de las nueve dimensiones que componen la experiencia óptima. Considerando la adecuación lingüística y sintáctica de los ítems se realizó una selección de los mismos en función del grado en que reflejaban un dominio específico de contenido del constructo que se pretendía medir.

A partir de ello, se obtuvo una escala inicialmente reducida de 24 ítems con cinco opciones de respuesta (desde "Totalmente de acuerdo" a "Totalmente en desacuerdo") que incluía de 2 a 3 ítems por cada una de las nueve dimensiones de la experiencia óptima (flow). A los adolescentes se les pedía que marquen el grado de acuerdo con cada uno de los ítems. Esta escala se administró a los adolescentes para evaluar las experiencias óptimas en las actividades curriculares complementarias, y realizar la selección de los 9 ítems definitivos.

Dado que el objetivo era lograr un instrumento válido y confiable de 9 reactivos que mida la experiencia óptima (flow) total no se asumió el criterio de realizar esta primera depuración por dimensión sino con el total de la escala. Al realizar el análisis de discriminación ítem- total corregido, se tomo el criterio estricto de eliminar aquellos reactivos con correlaciones inferiores a .40. En el análisis de homogeneidad se priorizó la configuración de ítems que maximizaban el valor del Alpha de Cronbach.

Una vez que los nueve ítems fueron seleccionados se procedió a realizar un análisis factorial exploratorio seguido de un análisis factorial confirmatorio para evaluar la estructura conceptual propuesta. El análisis factorial confirmatorio tiene como supuesto la normalidad de las variables, se asume que el critical ratio de los índices de asimetría y curtosis deben ser menores que |1.96| para que este supuesto se cumpla (Arbuckle, 2006; Byrne, 2010). Al analizar la distribución de las variables se transformaron a logaritmo natural aquellas variables que no cumplían con el supuesto de normalidad. Para probar el ajuste del modelo se utilizaron los siguientes índices: (1) RMR (Root Mean Residual); (2) RMSEA (Root Mean Square Error of Aproximation). Para estos indicadores se pueden considerar que valores iguales o inferiores a .08 indican un buen ajuste (Browne \& Cudeck, 1993). (3) GFI (Goodness of Fit Index); (4) AGFI (Adjusted Goodness of Fit Index), (5) CFI (Comparative Fit Index); (6) TLI (o índice de Tucker-Lewis). Para estos índices se consideran adecuados 
valores iguales o superiores a .90 (Hu \& Bentler, 1998, 1999; Shumacker \& Lomax, 1996). Se descarto el índice $\chi^{2}$ dado que pone a prueba la misma hipótesis nula referida al ajuste del modelo que los residuos, pero tiene desventajas como sus fuertes supuestos y una alta sensibilidad al tamaño muestral (García Cueto, Gallo Álvaro, \& Miranda, 1998; Martínez Arias, 1995).

Para la validez concurrente y discriminante se analizó el comportamiento de los ítems en relación al Cuestionario sobre el desarrollo de actividades (ad hoc). Este cuestionario permite evaluar la experiencia personal del adolescente al participar de alguna actividad.

La administración del instrumento definitivo se replicó en los mismos adolescentes para evaluar las experiencias óptimas en actividades extracurriculares (artísticas y deportivas realizadas por fuera de la institución educativa) y actividades sociales aportando mayores evidencias a la validez de la escala. Los casos con datos faltantes fueron eliminados para mantener una mayor rigurosidad en el análisis.

Se utilizaron los paquetes estadísticos SPSS y AMOS versión 18.

\section{Resultados.}

Análisis descriptivo de los ítems

A partir de administrar el inventario de experiencias óptimas en las actividades curriculares se alcanzó la estructura final de 9 ítems, correspondiendo cada uno de estos reactivos a una de las dimensiones planteadas por Csikszentmihalyi (1990, 1998).

Los 9 ítems que componen el inventario tienen cinco opciones de respuesta (“Totalmente de acuerdo"(1), "De acuerdo"(2), "Ni de acuerdo ni en desacuerdo"(3), "En desacuerdo"(4), "Totalmente en desacuerdo"(5)).: 1) Mientras realizo la actividad se me borran los problemas y las preocupaciones (Pérdida de la autoconciencia); 2) Tengo una buena idea, cuando estoy realizando la actividad, acerca de cuán bien lo estoy haciendo (Retroalimentación clara y directa); 3) Hago las cosas espontánea y automáticamente sin tener que pensarlas (Unión entre la acción y la conciencia); 4) Tengo una total concentración (Concentración en la tarea presente); 5)La manera en la que el tiempo pasa parece ser diferente de lo normal (Deformación en la percepción del tiempo); 6) Siento que soy lo suficientemente competente para alcanzar las demandas de la situación (Equilibrio entre la habilidad percibida y el desafío que plantea la actividad); 7) La experiencia es extremadamente gratificante (Experiencia autotélica); 8) Tengo un sentimiento de control 
total (Sentimiento o percepción de control); 9) Tengo un amplio sentido de lo que quiero hacer (Metas claras). En todos los reactivos se obtiene una puntuación directa de 1 a 5 que dan lugar al valor de experiencia óptima total que reporta el adolescente.

En la Tabla 1 puede observarse que todos los ítems del inventario, a excepción del segundo y el noveno, tienen curtosis negativa mostrando una distribución platicúrtica con una menor concentración de datos en torno a la media. El ítems 2 y 9 tiene una curtosis positiva mostrando una distribución leptocúrtica con una mayor concentración de los datos en torno a la media. Por otro lado, se observa una asimetría positiva indicando una predominancia de elección de valores bajos mostrando un mayor grado de acuerdo con los ítems, lo cual indicaría una tendencia a experimentar el estado óptimo.

Tabla 1. Análisis descriptivo de los items

\begin{tabular}{|c|c|c|c|c|c|c|c|c|c|c|c|c|c|c|c|}
\hline & \multirow[t]{3}{*}{ Media } & \multirow[t]{3}{*}{ D.E } & \multirow{3}{*}{$\begin{array}{l}\text { Curtosis } \\
\text { (E.T } \\
.333)\end{array}$} & \multirow{3}{*}{$\begin{array}{l}\text { Asimetría } \\
\text { (E.T .170) }\end{array}$} & \multirow{3}{*}{$\begin{array}{c}\text { Correlación } \\
\text { Ítem- total } \\
\text { corregida }\end{array}$} & \multicolumn{5}{|c|}{ Percentiles } & \multicolumn{5}{|c|}{ Respuestas \% } \\
\hline & & & & & & 10 & 25 & 50 & 75 & 90 & $1^{*}$ & $2 *$ & $3 *$ & $4^{*}$ & $5^{*}$ \\
\hline & & & & & & & & & & & & & & & \\
\hline Ítem 1 & 2.28 & 1.189 & -.459 & .669 & .620 & 1 & 1 & 2 & 3 & 4 & 31.8 & 30.8 & 20.4 & 11.4 & 5.7 \\
\hline Ítem 2 & 2.23 & .994 & .464 & .840 & .629 & 1 & 2 & 2 & 3 & 4 & 22.3 & 46.9 & 19.4 & 8.1 & 3.3 \\
\hline Ítem 3 & 2.69 & 1.189 & -.893 & .222 & .461 & 1 & 2 & 3 & 4 & 4 & 18 & 29.4 & 25.1 & 20.4 & 7.1 \\
\hline Ítem 4 & 2.27 & 1.178 & -.702 & .589 & .576 & 1 & 1 & 2 & 3 & 4 & 32.7 & 30.3 & 18 & 15.2 & 3.8 \\
\hline Ítem 5 & 2.37 & 1.169 & -.596 & .526 & .442 & 1 & 1 & 2 & 3 & 4 & 28 & 30.3 & 23.7 & 12.8 & 5.2 \\
\hline Ítem 6 & 2.38 & 1.108 & -.087 & .628 & .549 & 1 & 2 & 2 & 3 & 4 & 23.7 & 34.1 & 28.9 & 7.1 & 6.2 \\
\hline Ítem 7 & 2.40 & 1.119 & -.224 & .535 & .673 & 1 & 2 & 2 & 3 & 4 & 24.6 & 29.9 & 32.2 & 7.1 & 6.2 \\
\hline Ítem 8 & 2.50 & 1.119 & -.605 & .305 & .688 & 1 & 2 & 3 & 3 & 4 & 22.7 & 27 & 32. & 12.8 & 4.7 \\
\hline Ítem 9 & 2.09 & 1.061 & .404 & .939 & .741 & 1 & 1 & 2 & 3 & 4 & 34.1 & 37.4 & 18 & 6.6 & 3.8 \\
\hline Flow & 2.36 & .780 & .815 & .661 & & 1.36 & 1.67 & 2.33 & 2.78 & 3.22 & & & & & \\
\hline Total & & & & & & & & & & & & & & & \\
\hline
\end{tabular}

Nota: D.E: Desvío estándar. E.T: Error típico. "Totalmente de acuerdo"(1), "De acuerdo"(2), "Ni de acuerdo ni en desacuerdo"(3), "En desacuerdo"(4), "Totalmente en desacuerdo"(5)

Atendiendo a la validez del instrumento en el análisis de discriminación ítem total corregida se obtuvieron correlaciones por encima de .44.

\section{Fiabilidad del instrumento}

En el análisis de confiabilidad del inventario con la configuración final de 9 ítems se arribó a un Alpha de Cronbach aceptable de .864 .

\section{Análisis factorial}

El análisis factorial exploratorio (método de componentes principales) mostró una estructura compuesta por un único factor, sin forzar, que explica el $49.05 \%$ de la varianza 
total. Se obtuvo un excelente índice KMO igual a .901 y la prueba de esfericidad de Bartlett indicó que la matriz no era idéntica $\left(\chi^{2}=713.065, p<.001\right)$. Las saturaciones en el componente fueron desde .720 (ítem 1), .732 (ítem 2), .559 (ítem 3), .689 (ítem 4), 533 (ítem 5), .657 (ítem 6), .760 (ítem 7), .780 (ítem 8), 826 (ítem 9).

Se puso a prueba el modelo unifactorial en el que el factor latente satura a los 9 ítems de la escala mediante análisis factorial confirmatorio, utilizando el método de máxima verosimilitud. Se transformaron a logaritmo natural las variables que no cumplían con el supuesto de normalidad (Ítems: 1, 2, 4, 5, 6, 7 y 9). Los resultados mostraron excelentes índices de ajuste $(\mathrm{AGFI}=.90 ; \mathrm{GFI}=.94, \mathrm{TLI}=.95 ; \mathrm{CFI}=.96 ; \mathrm{RMSR}=.05 ; \mathrm{RMSEA}=0.07)$. En la Figura 1 se presenta el modelo con los pesos de regresión de las variables, que van desde .49 a .81 . Estos resultados aportan datos sobre la validez de constructo del inventario, de acuerdo a la estructura conceptual puesta a prueba.

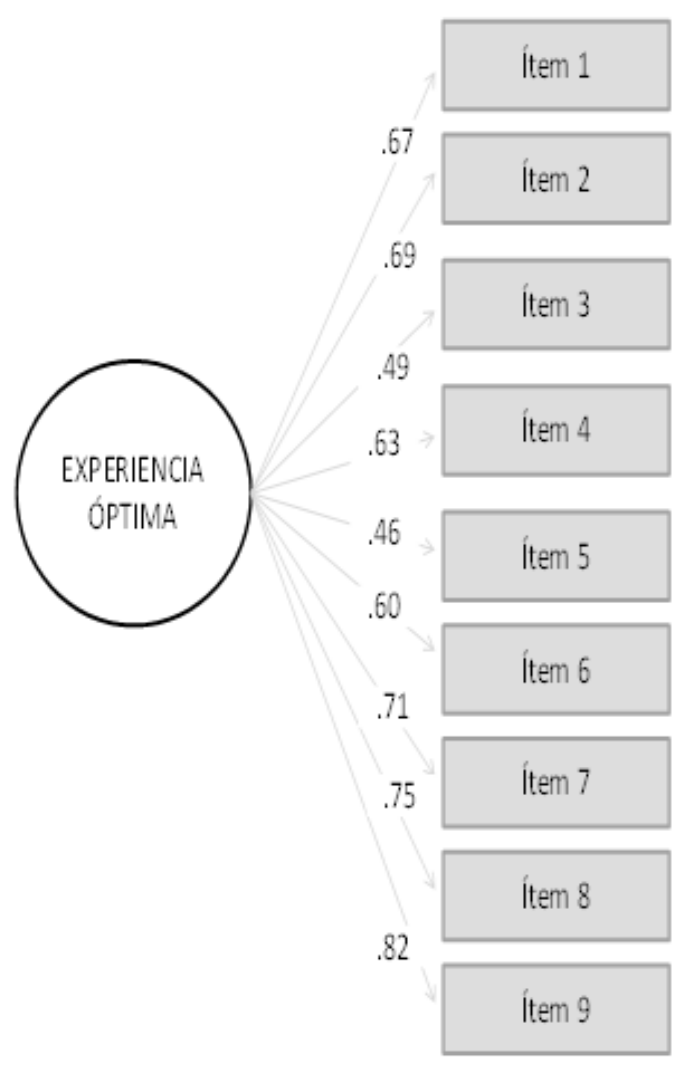


De acuerdo con los resultados obtenidos en el análisis factorial puede afirmarse que los 9 reactivos cargan para obtener una medida de experiencia óptima (flow) total.

\section{Replicación del inventario}

Luego se procedió a evaluar las experiencias óptimas de los mismos sujetos en las actividades extracurriculares (actividades artísticas y deportivas llevadas a cabo por fuera de la institución educativa) y las actividades sociales.

En ambas actividades se obtuvieron adecuados Alpha de Cronbach con correlaciones ítems total corregidas superiores .3 en todos los reactivos (tabla 2).

\begin{tabular}{|c|c|c|}
\hline & Actividades & Actividades \\
\hline & Extracurriculares & Sociales \\
\hline Ítem 1 & .60 & .49 \\
\hline Ítem 2 & .54 & .46 \\
\hline Ítem 3 & .38 & .31 \\
\hline Ítem 4 & .53 & .43 \\
\hline Ítem 5 & .35 & .49 \\
\hline Ítem 6 & .35 & .60 \\
\hline Ítem 7 & .48 & .59 \\
\hline Ítem 8 & .60 & .48 \\
\hline Ítem 9 & .54 & .54 \\
\hline Alfa de Cronbach & .79 & .79 \\
\hline
\end{tabular}

Al realizar el análisis factorial confirmatorio sobre los resultados obtenidos en las actividades extracurriculares y sociales con el inventario breve sobre experiencias óptimas se obtuvieron excelentes índices de ajuste del modelo unifactorial propuesto (Tabla 3). 
Tabla 3. Índices de ajustes del análisis factorial confirmatorio para cada actividad

\begin{tabular}{ccccccc}
\hline & TLI & SRMR & AGFI & CFI & RMSEA & GFI \\
\hline Curricular & .95 & .05 & .90 & .96 & 0.07 & .94 \\
Extracurricular & .935 & .0493 & .907 & .951 & .066 & 944 \\
Sociales & .914 & .051 & .908 & .935 & .075 & .945 \\
\hline
\end{tabular}

Finalmente no se hallaron diferencias significativas entre ambos sexos ( $p>.05)$, para ninguna de las tres actividades evaluadas.

\section{Validez convergente y discriminante}

Se administró un cuestionario, realizado ad hoc, que permite evaluar el estado subjetivo del adolescente al participar de alguna actividad. Es cuestionario estaba compuesto por seis afirmaciones con cinco opciones de respuesta (desde "Totalmente de acuerdo" hasta "Totalmente en desacuerdo"). Los ítems se agrupan en dos subescalas, una conformada por tres ítems que evalúan aspectos positivos de la experiencia en el desarrollo de actividades (ej. "Me divierte"), y tres que evalúan aspectos negativos de la misma (ej. "Me siento inseguro"). Se obtuvieron coeficientes Alpha de Cronbach de .86 y .72 respectivamente, para cada subescala.

Al realizar una correlación entre los índices de experiencia óptima obtenidos con el inventario en las actividades curriculares con los resultados obtenidos en el Cuestionario sobre el desarrollo de actividades se obtuvo una correlación positiva significativa (Rho de Spearman.694; $\mathrm{p}<.001)$ entre la experiencia óptima total y la subescala de experiencia positiva y una correlación negativa significativa (Rho de Spearman -.459; p<.001) con la subescala de vivencia negativa. Al repetir el análisis por ítems se mantuvo la relación explicada entre los componentes de ambos inventarios. Esto último aporta pruebas sobre la validez convergente y discriminante de la prueba.

\section{Discusión.}

El objetivo de este trabajo fue validar y presentar las propiedades psicométricas del inventario breve para evaluar las experiencias óptimas en adolescentes de nuestro contexto 
socio cultural.

Para ello se realizó un análisis discriminación de los ítems, se estimo la consistencia interna y la evidencia a favor de la validez concurrente y discriminante. Los resultados indican que todos los ítems cuentan con una alta capacidad discriminativa y que presentan una adecuada homogeneidad. Por otro lado, las correlaciones observadas con la medida externa utilizada (Cuestionario sobre el desarrollo de actividades) muestran evidencias a favor de la validez concurrente y discriminante.

En análisis factorial exploratorio se obtuvo, sin forzar los datos, una estructura formada por un solo factor latente. Los índices obtenidos en el análisis factorial confirmatorio para el modelo unifactorial en el que el factor latente satura a los 9 ítems, fueron adecuados en todas las actividades.

Todos los resultados obtenidos brindan información sobre una adecuada validez y confiabilidad del inventario de experiencias óptimas en adolescentes del área metropolitana de Buenos Aires. Por otro lado, el inventario mostró ser válido y confiable para su aplicación en diversas actividades en que los adolescentes participan.

El inventario breve sobre experiencias óptimas, sobre el cual se trabajo, está compuesto por nueve ítems cada uno de los cuales representa una de las nueve dimensiones propuestas por Csikszentmihalyi (1990, 1998). Los resultados obtenidos, en relación a la validez de la estructura conceptual con un único factor latente, son congruentes con los hallados por Jackson y Col. (2008) en su versión reducida de la Flow Scale. Asimismo, considerando que cada uno de los ítems corresponde a una de las nueve dimensiones o características de la experiencia óptima, la estructura puesta a prueba aporta datos sobre la validez del modelo conceptual que plantea una composición multidimensional de la experiencia óptima (Csikszentmihalyi, 1990; Csikszentmihalyi \& Csikszentmihalyi, 1998; Jackson et al., 2008).

La importancia de validar un instrumento autoinformado que evalúa las experiencias óptimas no solo en una sino en varias actividades en que los adolescentes de nuestro medio participan reside en poder crear un instrumento con buenos índices de confiabilidad y ecológicamente válido (Leibovich, Schufer \& Muiños, 1998).

Asimismo, contar con un inventario breve para evaluar las experiencias óptimas en adolescentes resulta útil contemplando situaciones en que administrar una versión más extensa puede resulta dificultoso (Jackson et al., 2008). 
Se plantea como necesidad en futuras investigaciones: probar la validez y la confiabilidad del inventario en otras actividades adolescentes distintas a las evaluadas en el presente trabajo; e indagar la relación de este constructo con variables asociadas al bienestar personal para conocer el alcance de los efectos de las experiencias óptimas en el desarrollo del individuo durante esta franja etaria.

\section{Referencias}

Arbuckle, J. L. (2006). AMOS 7.0. User's Guide. Chicago, IL: Smallwaters Corporation.

Bakker, A. B. (2005). Flow among music teachers and their students: The crossover of peak experiences. Journal of Vocational Behavior 66, 26-44.

Bakker, A. B. (2008). The work-related flow inventory: Construction and initial validation of the WOLF Journal of Vocational Behavior, 72(3), 400-414.

Browne, M. W., \& Cudeck, R. (1993). Alternative way of assessing model fit. In K. A. Bollen \& J. S. Long (Eds.), Testing Structural Equation Models (pp. 8136-8162): Newbury Park: Sage Publication.

Byrne, B. (2010). Structural equation modeling with AMOS. Basic concepts, applications, and programming (2a. ed.). New York: Routledge.

Csikszentmihalyi, M. (1990). Fluir. Una psicología de la felicidad. Barcelona: Editorial Kairós.

Csikszentmihalyi, M., \& Csikszentmihalyi, I. (1998). Experiencia óptima. Estudios psicológicos del flujo en la conciencia: Bilbao: Desclée de Brouwer.

Csikszentmihalyi, M., \& Larson, R. W. (1984). Being adolescent. New York.

Csikszentmihalyi, M., Larson, R. W., \& Prescott, S. (1977). The Ecology of Adolescent Activity and Experience. Journal of Youth and Adolescence,, 6(3), 181-294.

Csikszentmihalyi, M., \& LeFevre, J. (1989). Optimal Experience in Work and Leisure. Journal of Personality and Social Psychology, 56(5), 815-822.

Chu, L.-C. (2010). Flow Experience of Knowledge Workers: A Case Study of a Taiwanese Consultancy. The Journal of International Management Studies, 5(2), 216-226.

Finneran, C. M., \& Zhang, P. (2005). Flow in Computer- Mediated Environments: Promises and Challenges. Communications of the Association for Information Systems 15, 82 101.

Fournier, J., Gaudreau, P., Demontrond-Behr, P., Visioli, J., Forest, J., \& Jackson, S. (2007). French translation of the Flow State Scale-2: Factor structure, cross-cultural invariance, and associations with goal attainment. Psychology of sport and exercise, 8(6), 897-916. 
Fritz, B. S., \& Avsec, A. (2007). The experience of flow and subjective well-being of music students. 16, 2, 5-17.

García Calvo, T., Jiménez Castuera, R., Santos-Rosa Ruano, F. J., Reina Vaíllo, R., \& Cervelló Gimeno, E. (2008). Psychometric Properties of the Spanish Version of the Flow State Scale. The Spanish Journal of Psychology 11(2), 660-669.

García Cueto, E., Gallo Álvaro, P., \& Miranda, R. (1998). Bondad de ajuste en el análisis factorial confirmatorio. Psicothema, 717-723.

García, T., Cervelló, E. M., Jiménez, R., Iglesias, D., \& Santos-Rosa, F. J. (2005). La implicación motivacional de jugadores jóvenes de fútbol y su relación con el estado de flow y la satisfacción en competición. Revista de Psicología del Deporte, 14(1), 21-42.

Ghani, J. A., \& Deshpande, S. P. (1994). Task characteristics and the experience of optimal flow in human-computer interaction. The joumal of Psychology, 128, 381-391.

Gouveia, M. J., Pais-Ribeiro, J. L., Moreira Marques, M., \& Carvalho, C. M. (2012). Validity and Reliability of the Portuguese Version of the Dispositional Flow Scale-2 in Exercise. Revista de Psicología del Deporte, 21(1), 81-88.

Hu, L., \& Bentler, P. M. (1998). Fit indices in covariance structure modeling: Sensitivity to underparameterized model misspecification. . Psychological Methods, 3, 424-453.

Hu, L., \& Bentler, P. M. (1999). Cut-off criteria for fit indexes in covariance structure analysis: Conventional criteria versus new alternatives. Structural Equation Modeling, $6,1-55$.

Jackson, S., \& Marsh, H. W. (1996). Development and validation of a scale to measure optimal experience: The Flow State Scale. Journal of Sport \& Exercise Psychology, 18(1), 17-35.

Jackson, S., Martin, A., \& Eklund, R. (2008). Long and Short Measures of Flow: The Construct Validity of the FSS-2, DFS-2, and New Brief Counterparts. Journal of Sport and Exercise Psychology, 30, 561-587.

Jackson, S. A., \& Eklund, R. C. (2002). Assessing flow in physical activity: The Flow State Scale-2 and Dispositional Flow State Scale-2. Journal of Sport and Exercise Psychology, 24, 115-133.

Jackson, S. A., Kimiecik, J. C., Ford, S. K., \& Marsh, H. W. (1998). Psychological Correlates of Flow in Sport. Journal of Sport \& Exercise Psychology, 20, 358-378

Jackson, S. A., Thomas, P. R., Marsh, H. W., \& Smethurst, C. J. (2001). Relationships between Flow, Self-Concept, Psychological Skills, and Performance. Jounal of Applied Sport Psychology, 13, 129-133.

Keller, J. (2008). Flow and Regulatory Compatibility: An Experimental Approach to the Flow Model of Intrinsic Motivation. . Pers. Soc. Psychol. Bull, 34(2), 196-209. 
Kimiecik, J. C., \& Jackson, S. A. . (2002). Optimal experience in sport: A flow perspective. In I. T. Horn (Ed.), Advances in sport psychology. Champaign IL: Human Kinetics. Leibovich, N. (2009). El concepto de flow (fluir) en el trabajo. Aristeo, 1(1), 93-106.

Leibovich, N.; Schufer, M. \& Muiños, R. (1998). Ecoevaluación psicológica. Las figuras humanas en contexto familiar y escolar. Buenos Aires: Eudeba

López Torres, M., Torregosa, M. S., \& Roca, J. (2007). Características y Relaciones de "Flow", Ansiedad y Estado Emocional con el Rendimiento Deportivo en Deportistas de Elite. . Cuadernos de Psicología del Deporte, 7(1).

Martin, A. J., Liem, G. A., Coffey, 1., Martinez, C., Parker, P. P., Marsh, H. W., \& Jackson, S. A. (2010). What Happens to Physical Activity Behavior, Motivation, Self-Concept, and Flow After Completing School? A Longitudinal Study. Journal of Applied Sport Psychology, 22, 437- 457.

Martínez Arias, R. (1995). Psicometría: Teoría de los tests psicológicos y educativos.: Madrid: Síntesis.

Mesurado, B. (2008). Validez Factorial y Fiabilidad del Cuestionario de Experiencia Óptima (Flow) para niños y adolescentes. RIDEP 1(25), 159 - 178.

Moreno, J. A., Alonso, N., Martínez Galindo, C., \& Cervelló, E. (2005). Motivación, disciplina, coeducación y estado de flow en educación

física: Diferencias según la satisfacción, la práctica deportiva y la frecuencia de práctica. Cuadernos de Psicología del Deporte, 5(1-2), 225-243.

Moreno Murcia, J. A., Cervelló Gimeno, E., \& González-Cutre, D. (2008). Relationships among Goal Orientations, Motivational Climate

and Flow in Adolescent Athletes: Differences by Gender. The Spanish Journal of Psychology, 11(1), 181-191.

Roberts, B. W., Walton, K. E., \& Bogg, T. (2005). Conscientiousness and health across the life course. Review of General Psychology, 9, 156-168.

Rodríguez-Sánchez, A. M., Schaufeli, W. B., Salanova, M., \& Cifre, E. (2008). Flow experience among information and communication technology users. Psychological Reports, 102, 29-39.

Salanova, M., Bakker, A. B., \& Llorens, S. (2006). Flow at Work: Evidence for an Upward Spiral of Personal and Organizational Resources. Journal of Happiness Studies, 7, 122.

Salanova, M., Martínez, I. M., Cifre, E., \& Schaufeli, W. B. (2005). ¿Se pueden ivvir experiencias óptimas en el trabajo? Analizando el flow en contextos laborales. Revista de psicología general y aplicada, 58(1), 89-100.

Seifert, T., \& Hedderson, C. (2010). Intrinsic Motivation and Flow in Skateboarding: An 
Ethnographic Study. Journal of Happiness Studies, 11(3), 277-292.

Seligman, M., \& Csikszentmihalyi, M. (2000). Positive Psychology: An Introduction. American Psychologist, 55(1), 5-14.

Shernoff, D. J., Csikszentmihalyi, M., Schneider, B., \& Shernoff, E. S. (2003). Student engagement in high school classrooms from the perspective of flow theory. . School Psychology Quarterly, 18(2), 158-176.

Shernoff, D. J., \& Vandell, D. L. (2007). Engagement in after-school program activities: quality of experience from the perspective of participants. J Youth Adolescence, 36, 891-903.

Shumacker, R. E., \& Lomax, R. G. (1996). A beginner guide to structural equation modeling: Mahwah: Erlbaum. 